\title{
A quantum long time energy red shift: a contribution to varying $\alpha$ theories
}

\author{
K. Urbanowski* \\ University of Zielona Góra, Institute of Physics, \\ ul. Prof. Z. Szafrana 4a, 65-516 Zielona Góra, Poland.
}

July 14, 2018

\begin{abstract}
By analyzing the survival probability amplitude of an unstable state we show that the energy corrections to this state in the long $(t \rightarrow \infty)$ and relatively short (lifetime of the state) time regions, are different. It is shown that in the considered model the above corrections decrease to zero as $t \rightarrow \infty$. It is hypothesized that this property could be detected by analyzing the spectra of distant astrophysical objects. The above property of unstable states may influence the measured values of possible deviations of the fine structure constant $\alpha$ as well as other astrophysical and cosmological parameters.
\end{abstract}

PACS numbers: 03.65.-w, 11.10.St, 06.20.Jr, 98.62.Py

Keywords: unstable states, nonexponential decay, red shift, varying alpha.

\section{Introduction}

Within the quantum theory the state vector at time $t,|\Phi(t)\rangle$, for the physical system under consideration which initially (at $t=t_{0}=0$ ) was in the state

*e-mail: K.Urbanowski@proton.if.uz.zgora.pl; K.Urbanowski@if.uz.zgora.pl 
$|\Phi\rangle$ can be found by solving the Schödinger equation

$$
i \hbar \frac{\partial}{\partial t}|\Phi(t)\rangle=H|\Phi(t)\rangle, \quad|\Phi(0)\rangle=|\Phi\rangle,
$$

where $|\Phi(t)\rangle,|\Phi\rangle \in \mathcal{H}, \mathcal{H}$ is the Hilbert space of states of the considered system, $\||\Phi(t)\rangle\|=\||\Phi\rangle \|=1$ and $H$ denotes the total selfadjoint Hamiltonian for the system. If one considers an unstable state $|\Phi\rangle \equiv|\phi\rangle$ of the system then using the solution $|\phi(t)\rangle$ of Eq. (11) for the initial condition $|\phi(0)\rangle=|\phi\rangle$ one can determine the decay law, $\mathcal{P}_{\phi}(t)$ of this state decaying in vacuum

$$
\mathcal{P}_{\phi}(t)=|a(t)|^{2}
$$

where $a(t)$ is probability amplitude of finding the system at the time $t$ in the initial state $|\phi\rangle$ prepared at time $t_{0}=0$,

$$
a(t)=\langle\phi \mid \phi(t)\rangle \text {. }
$$

We have

$$
a(0)=1
$$

From basic principles of quantum theory it is known that the amplitude $a(t)$, and thus the decay law $\mathcal{P}_{\phi}(t)$ of the unstable state $|\phi\rangle$, are completely determined by the density of the energy distribution $\omega(\mathcal{E})$ for the system in this state [1],

$$
a(t)=\int_{\text {Spec. }(H)} \omega(\mathcal{E}) e^{-\frac{i}{\hbar} \mathcal{E} t} d \mathcal{E} .
$$

where $\omega(\mathcal{E})>0$.

Note that (5) and (4) mean that there must be

$$
a(0)=\int_{\text {Spec. }(H)} \omega(\mathcal{E}) d \mathcal{E}=1
$$

From the last property and from the Riemann-Lebesgue Lemma it follows that the amplitude $a(t)$, being the Fourier transform of $\omega(\mathcal{E})$ (see (5) ), must tend to zero as $t \rightarrow \infty[1]$.

In [2] assuming that the spectrum of $H$ must be bounded from below, (Spec. $(H)>-\infty)$, and using the Paley-Wiener Theorem [3] it was proved that in the case of unstable states there must be

$$
|a(t)| \geq A e^{-b t^{q}}
$$


for $|t| \rightarrow \infty$. Here $A>0, b>0$ and $0<q<1$. This means that the decay law $\mathcal{P}_{\phi}(t)$ of unstable states decaying in the vacuum, (2), can not be described by an exponential function of time $t$ if time $t$ is suitably long, $t \rightarrow \infty$, and that for these lengths of time $\mathcal{P}_{\phi}(t)$ tends to zero as $t \rightarrow \infty$ more slowly than any exponential function of $t$. The analysis of the models of the decay processes shows that $\mathcal{P}_{\phi}(t) \simeq e^{-\frac{\gamma_{\phi}^{0} t}{\hbar}}$, (where $\gamma_{\phi}^{0}$ is the decay rate of the state $|\phi\rangle)$, to an very high accuracy for a wide time range $t$ : From $t$ suitably latter than some $T_{0} \simeq t_{0}=0$ but $T_{0}>t_{0}$ up to $t \gg \tau_{\phi}=\frac{\gamma_{\phi}^{0}}{\hbar}$ and smaller than $t=T_{\infty}$, where $T_{\infty}$ denotes the time $t$ for which the nonexponential deviations of $a(t)$ begin to dominate (see eg., [2], [4] - [8]). From this analysis it follows that in the general case the decay law $\mathcal{P}_{\phi}(t)$ takes the inverse power-like form $t^{-\lambda}$, (where $\lambda>0$ ), for suitably large $t \geq T_{\infty} \gg \tau_{\phi}$ [2], [4] - 7]. This effect is in agreement with the general result (7). Effects of this type are sometimes called the "Khalfin effect" (see eg. 9]).

Note that one can force $\mathcal{P}_{\phi}(t)$ to have an exponential form for all $t$ including $t \rightarrow \infty$ by using the time dependent decay rate [9, 10].

Unfortunately, there is no experimental evidence for the Khalfin effect. The performed test of the decay laws did not indicate deviations from the exponential form of $\mathcal{P}_{\phi}(t)$ at the long time region [11, 12, 8], neither did they show a time dependence of the decay rate at this time region. Following the estimations given in [5] one infers that this probably occurs because it is almost impossible to build in a laboratory a device detecting decays of the unstable states in the vacuum which allows one to observe these decays from the instant $t=t_{0}=0$ of the preparation of these states up to times $t \sim 10^{n} \tau_{\phi}$, where $n \geq 9$.

Note that in fact the amplitude $a(t)$ contains information about the decay law $\mathcal{P}_{\phi}(t)$ of the state $|\phi\rangle$, that is about the decay rate $\gamma_{\phi}^{0}$ of this state, as well as the energy $\mathcal{E}_{\phi}^{0}$ of the system in this state. This information can be extracted from $a(t)$. Indeed if $|\phi\rangle$ is an unstable (a quasi-stationary) state then

$$
a(t) \cong e^{-\frac{i}{\hbar}\left(\mathcal{E}_{\phi}^{0}-\frac{i}{2} \gamma_{\phi}^{0}\right) t}
$$

So, there is

$$
\mathcal{E}_{\phi}^{0}-\frac{i}{2} \gamma_{\phi}^{0} \equiv i \hbar \frac{\partial a(t)}{\partial t} \frac{1}{a(t)}
$$

in the case of quasi-stationary states. 
The standard interpretation and understanding of the quantum theory and the related construction of our measuring devices are such that detecting the energy $\mathcal{E}_{\phi}^{0}$ and decay rate $\gamma_{\phi}^{0}$ one is sure that the amplitude $a(t)$ has the form (8) and thus that the relation (9) occurs. Taking the above into account one can define the "effective Hamiltonian", $h_{\phi}$, for the one-dimensional subspace of states $\mathcal{H}_{\|}$spanned by the normalized vector $|\phi\rangle$ as follows (see, eg. [10])

$$
h_{\phi} \stackrel{\text { def }}{=} i \hbar \frac{\partial a(t)}{\partial t} \frac{1}{a(t)} .
$$

In general, $h_{\phi}$ can depend on time $t, h_{\phi} \equiv h_{\phi}(t)$. One meets this effective Hamiltonian when one starts with the Schrödinger Equation (1) for the total state space $\mathcal{H}$ and looks for the rigorous evolution equation for the distinguished subspace of states $\mathcal{H}_{\|} \subset \mathcal{H}$. In the case of one-dimensional $\mathcal{H}_{\|}$this rigorous Schrödinger-like evolution equation has the following form for the initial condition $a(0)=1$ (see [10] and references one finds therein),

$$
i \hbar \frac{\partial a(t)}{\partial t}=h_{\phi}(t) a(t)
$$

Relations (10) and (11) establish a direct connection between the amplitude $a(t)$ for the state $|\phi\rangle$ and the exact effective Hamiltonian $h_{\phi}(t)$ governing the time evolution in the one-dimensional subspace $\mathcal{H}_{\|} \ni|\phi\rangle$. Thus the use of the evolution equation (11) or the relation (10) is one of the most effective tools for the accurate analysis of the early- as well as the long-time properties of the energy and decay rate of a given qausistationary state $|\phi(t)\rangle$.

So let us assume that we know the amplitude $a(t)$. Then starting with this $a(t)$ and using the expression (10) one can calculate the effective Hamiltonian $h_{\phi}(t)$ in a general case for every $t$. Thus, one finds the following expressions for the energy and the decay rate of the system in the state $|\phi\rangle$ under considerations,

$$
\begin{aligned}
\mathcal{E}_{\phi} & \equiv \mathcal{E}_{\phi}(t)=\Re\left(h_{\phi}(t),\right. \\
\gamma_{\phi} & \equiv \gamma_{\phi}(t)=-2 \Im\left(h_{\phi}(t),\right.
\end{aligned}
$$

where $\Re(z)$ and $\Im(z)$ denote the real and imaginary parts of $z$ respectively.

As it was mentioned above the deviations of the decay law $\mathcal{P}_{\phi}(t)$ from the exponential form can be described equivalently using time-dependent decay rate. In terms of such $\gamma_{\phi}(t)$ the Khalfin observation that $\mathcal{P}_{\phi}(t)$ must tend 
to zero as $t \rightarrow \infty$ more slowly than any exponential function means that $\gamma_{\phi}(t) \ll \gamma_{\phi}^{0}$ for $t \gg T_{\infty}$ and $\lim _{t \rightarrow \infty} \gamma_{\phi}(t)=0$.

The aim of this note is to examine the long time behaviour of $\mathcal{E}_{\phi}(t)$ and $\gamma_{\phi}(t)$ using $a(t)$ calculated for the given density $\omega(\mathcal{E})$. We show that $\mathcal{E}_{\phi}(t) \rightarrow 0$ as $t \rightarrow \infty$ for the model considered and that a wide class of models has similar long time properties: $\left.\mathcal{E}_{\phi}(t)\right|_{t \rightarrow \infty} \neq \mathcal{E}_{\phi}^{0}$. It seems that in contrast to the standard Khalfin effect [2] in the case of the quasistationary states belonging to the same class as excited atomic levels, this long time properties of the energy $\mathcal{E}_{\phi}(t)$ have a chance to be detected by analyzing spectra of very distant stars.

The paper is organized as follows. In Sec. 2 the above mentioned model is considered. Sec. 3 contains an analysis of some general case of $a(t)$ causing

$\mathcal{E}_{\phi}(t)$ to tend to zero as $t \rightarrow \infty$. A discussion and final remarks can be found in Sec. 4.

\section{The model}

Let us assume that $\operatorname{Spec} .(H)=[0, \infty)$ and let us choose $\omega(\mathcal{E})$ as follows

$$
\omega(\mathcal{E})=\frac{N}{2 \pi} \Theta(\mathcal{E}) \frac{\gamma_{\phi}^{0}}{\left(\mathcal{E}-\mathcal{E}_{\phi}^{0}\right)^{2}+\left(\frac{\gamma_{\phi}^{0}}{2}\right)^{2}},
$$

where $N$ is a normalization constant and

$$
\Theta(\mathcal{E})=\left\{\begin{array}{l}
1 \text { for } \mathcal{E} \geq 0 \\
0 \text { for } \mathcal{E}<0
\end{array}\right.
$$

For such $\omega(\mathcal{E})$ using (5) one has

$$
a(t)=\frac{N}{2 \pi} \int_{0}^{\infty} \frac{\gamma_{\phi}^{0}}{\left(\mathcal{E}-\mathcal{E}_{\phi}^{0}\right)^{2}+\left(\frac{\gamma_{\phi}^{0}}{2}\right)^{2}} e^{-\frac{i}{\hbar} \mathcal{E} t} d \mathcal{E},
$$

where

$$
\frac{1}{N}=\frac{1}{2 \pi} \int_{0}^{\infty} \frac{\gamma_{\phi}^{0}}{\left(\mathcal{E}-\mathcal{E}_{\phi}^{0}\right)^{2}+\left(\frac{\gamma_{\phi}^{0}}{2}\right)^{2}} d \mathcal{E} .
$$

Formula (15) leads to the result

$$
a(t)=N e^{-\frac{i}{\hbar}\left(\mathcal{E}_{\phi}^{0}-i \frac{\gamma_{\phi}^{0}}{2}\right) t} \times
$$




$$
\begin{gathered}
\times\left\{1-\frac{i}{2 \pi}\left[e^{\frac{\gamma_{\phi}^{0} t}{\hbar}} E_{1}\left(-\frac{i}{\hbar}\left(\mathcal{E}_{\phi}^{0}+\frac{i}{2} \gamma_{\phi}^{0}\right) t\right)\right.\right. \\
\left.\left.+(-1) E_{1}\left(-\frac{i}{\hbar}\left(\mathcal{E}_{\phi}^{0}-\frac{i}{2} \gamma_{\phi}^{0}\right) t\right)\right]\right\},
\end{gathered}
$$

where $E_{1}(x)$ denotes the integral-exponential function [13, 14].

Using (15) or (17) one easily finds that

$$
i \hbar \frac{\partial a(t)}{\partial t}=\left(\mathcal{E}_{\phi}^{0}-\frac{i}{2} \gamma_{\phi}^{0}\right) a(t)+\Delta a(t)
$$

where

$$
\begin{aligned}
\Delta a(t)=\frac{N}{\pi} \frac{\gamma_{\phi}^{0}}{2} & e^{-\frac{i}{\hbar}\left(\mathcal{E}_{\phi}^{0}+\frac{i}{2} \gamma_{\phi}^{0}\right) t} \times \\
& \times E_{1}\left(-\frac{i}{\hbar}\left(\mathcal{E}_{\phi}^{0}+\frac{i}{2} \gamma_{\phi}^{0}\right) t\right) .
\end{aligned}
$$

So,

$$
h_{\phi}(t) \equiv i \hbar \frac{\partial a(t)}{\partial t} \frac{1}{a(t)} \stackrel{\text { def }}{=} h_{\phi}^{0}+\Delta h_{\phi}(t),
$$

where

$$
h_{\phi}^{0} \equiv \mathcal{E}_{\phi}^{0}-\frac{i}{2} \gamma_{\phi}^{0}
$$

and

$$
\Delta h_{\phi}(t)=+\frac{\Delta a(t)}{a(t)}
$$

Using the asymptotic expansion of $E_{1}(x)$ [14],

$$
\left.E_{1}(z)\right|_{|z| \rightarrow \infty} \sim \frac{e^{-z}}{z}\left(1-\frac{1}{z}+\frac{2}{z^{2}}-\ldots\right)
$$

where $|\arg z|<\frac{3}{2} \pi$, one finds

$$
\begin{aligned}
\left.a(t)\right|_{t \rightarrow \infty} \simeq & N e^{-\frac{i}{\hbar} h_{\phi}^{0} t} \\
& +(-i) \frac{N}{2 \pi} \frac{\gamma_{\phi}^{0}}{\left|h_{\phi}^{0}\right|^{2}} \frac{\hbar}{t} \\
& +(-2) \frac{N}{2 \pi} \frac{\mathcal{E}_{\phi}^{0} \gamma_{\phi}^{0}}{\left|h_{\phi}^{0}\right|^{4}}\left(\frac{\hbar}{t}\right)^{2}+\ldots,
\end{aligned}
$$


and

$$
\begin{aligned}
\left.\Delta a(t)\right|_{t \rightarrow \infty} \simeq & i \frac{N \gamma_{\phi}^{0}}{2 \pi} \frac{h_{\phi}^{0}}{\left|h_{\phi}^{0}\right|^{2}} \frac{\hbar}{t} \\
& +\frac{N \gamma_{\phi}^{0}}{2 \pi} \frac{\left(h_{\phi}^{0}\right)^{2}}{\left|h_{\phi}^{0}\right|^{4}}\left(\frac{\hbar}{t}\right)^{2}+\ldots .
\end{aligned}
$$

These two last asymptotic expansions enable one to find (see (22) )

$$
\begin{aligned}
\left.\Delta h_{\phi}(t)\right|_{t \rightarrow \infty} & =\left.\frac{\Delta a(t)}{a(t)}\right|_{t \rightarrow \infty} \\
& \simeq-h_{\phi}^{0}-i \frac{\hbar}{t}-2 \frac{\mathcal{E}_{\phi}^{0}}{\left|h_{\phi}^{0}\right|^{2}}\left(\frac{\hbar}{t}\right)^{2}+\ldots .
\end{aligned}
$$

Thus, there is

$$
\left.h_{\phi}(t)\right|_{t \rightarrow \infty} \simeq-i \frac{\hbar}{t}-2 \frac{\mathcal{E}_{\phi}^{0}}{\left|h_{\phi}^{0}\right|^{2}}\left(\frac{\hbar}{t}\right)^{2}+\ldots
$$

for the considered case (14) of $\omega(\mathcal{E})$.

From (27) it follows that

$$
\Re\left(\left.h_{\phi}(t)\right|_{t \rightarrow \infty}\right) \stackrel{\text { def }}{=} \mathcal{E}_{\phi}^{\infty} \simeq-2 \frac{\mathcal{E}_{\phi}^{0}}{\left|h_{\phi}^{0}\right|^{2}}\left(\frac{\hbar}{t}\right)^{2} \longrightarrow 0,
$$

as $t \rightarrow \infty$, where $\mathcal{E}_{\phi}^{\infty}=\left.\mathcal{E}_{\phi}(t)\right|_{t \rightarrow \infty}$, and

$$
\Im\left(\left.h_{\phi}(t)\right|_{t \rightarrow \infty}\right) \simeq-\frac{\hbar}{t} \longrightarrow 0 \quad(\text { as } \quad t \rightarrow \infty) .
$$

For different states $\left|\phi_{1}\right\rangle,\left|\phi_{2}\right\rangle$ one has

$$
\begin{aligned}
\mathcal{E}_{\phi_{1}}^{\infty}-\mathcal{E}_{\phi_{2}}^{\infty} & =-2\left[\frac{\mathcal{E}_{\phi_{1}}^{0}}{\left|h_{\phi_{1}}^{0}\right|^{2}}-\frac{\mathcal{E}_{\phi_{2}}^{0}}{\left|h_{\phi_{2}}^{0}\right|^{2}}\right]\left(\frac{\hbar}{t}\right)^{2} \\
& \neq \mathcal{E}_{\phi_{1}}^{0}-\mathcal{E}_{\phi_{2}}^{0} \neq 0
\end{aligned}
$$

and

$$
\Im\left(\left.h_{\phi_{1}}(t)\right|_{t \rightarrow \infty}\right)=\Im\left(\left.h_{\phi_{2}}(t)\right|_{t \rightarrow \infty}\right),
$$

whereas in general $\gamma_{\phi_{1}} \neq \gamma_{\phi_{1}}$. 
The other relations are the following

$$
\begin{aligned}
& \frac{\mathcal{E}_{\phi_{1}}^{\infty}}{\mathcal{E}_{\phi_{2}}^{\infty}}=\frac{\mathcal{E}_{\phi_{1}}^{0}}{\mathcal{E}_{\phi_{2}}^{0}} \frac{\left|h_{\phi_{2}}^{0}\right|^{2}}{\left|h_{\phi_{1}}^{0}\right|^{2}} \\
&=\frac{\mathcal{E}_{\phi_{2}}^{0}}{\mathcal{E}_{\phi_{1}}^{0}} \frac{1+\frac{1}{4}\left(\frac{\gamma_{\phi_{2}}^{0}}{\mathcal{E}_{\phi_{2}}^{0}}\right)^{2}}{1+\frac{1}{4}\left(\frac{\gamma_{\phi_{1}}^{0}}{\mathcal{E}_{\phi_{1}}^{0}}\right)^{2}} \neq \frac{\mathcal{E}_{\phi_{1}}^{0}}{\mathcal{E}_{\phi_{2}}^{0}} \\
& \frac{\mathcal{E}_{\phi_{1}}^{\infty}-\mathcal{E}_{\phi_{2}}^{\infty}}{\mathcal{E}_{\phi_{1}}^{\infty}+\mathcal{E}_{\phi_{2}}^{\infty}}=\frac{\frac{\mathcal{E}_{\phi_{1}}^{0}}{\left|h_{\phi_{1}}^{0}\right|^{2}}-\frac{\mathcal{E}_{\phi_{2}}^{0}}{\left|h_{\phi_{2}}^{0}\right|^{2}}}{\frac{\mathcal{E}_{\phi_{1}}}{\left|h_{\phi_{1}}^{0}\right|^{2}}+\frac{\mathcal{E}_{\phi_{2}}^{0}}{\left|h_{\phi_{2}}^{0}\right|^{2}}} \\
& \equiv \frac{\mathcal{E}_{\phi_{1}}^{0}\left|h_{\phi_{2}}^{0}\right|^{2}-\mathcal{E}_{\phi_{2}}^{0}\left|h_{\phi_{1}}^{0}\right|^{2}}{\mathcal{E}_{\phi_{1}}^{0}\left|h_{\phi_{2}}^{0}\right|^{2}+\mathcal{E}_{\phi_{2}}^{0}\left|h_{\phi_{1}}^{0}\right|^{2}} \\
& \neq \frac{\mathcal{E}_{\phi_{1}}^{0}-\mathcal{E}_{\phi_{2}}^{0}}{\mathcal{E}_{\phi_{1}}^{0}+\mathcal{E}_{\phi_{2}}^{0}},
\end{aligned}
$$

and

$$
\frac{\Im\left(\left.h_{\phi_{1}}(t)\right|_{t \rightarrow \infty}\right)}{\Im\left(\left.h_{\phi_{2}}(t)\right|_{t \rightarrow \infty}\right)}=1 \neq \frac{\gamma_{\phi_{1}}^{0}}{\gamma_{\phi_{2}}^{0}} .
$$

It seems interesting that relations $(\underline{32})$ - (34) do not depend on time $t$.

Note that the following conclusion can be drawn from (30): For suitably long times $t$ there must be

$$
\left|\mathcal{E}_{\phi_{1}}^{\infty}-\mathcal{E}_{\phi_{2}}^{\infty}\right|<\left|\mathcal{E}_{\phi_{1}}^{0}-\mathcal{E}_{\phi_{2}}^{0}\right| .
$$

These suitable times can be estimated using relation (24). From (24) one obtains

$$
\left.|a(t)|_{t \rightarrow \infty}\right|^{2} \simeq N^{2} e^{-\frac{\gamma_{\phi}^{0}}{\hbar} t}+\frac{N^{2}}{4 \pi^{2}} \frac{\left(\gamma_{\phi}^{0}\right)^{2}}{\left|h_{\phi}^{0}\right|^{4}} \frac{\hbar^{2}}{t^{2}}+\ldots .
$$

Relations (26) - (35) become important for times $t>t_{a s}$, where $t_{a s}$ denotes the time $t$ at which contributions to $\left.|a(t)|_{t \rightarrow \infty}\right|^{2}$ from the first exponential component in (36) and from the second component proportional to $\frac{1}{t^{2}}$ are comparable. So $t_{a s}$ can be be found by considering the following relation

$$
e^{-\frac{\gamma_{\phi}^{0}}{\hbar} t} \sim \frac{1}{4 \pi^{2}} \frac{\left(\gamma_{\phi}^{0}\right)^{2}}{\left|h_{\phi}^{0}\right|^{4}} \frac{\hbar^{2}}{t^{2}}
$$


Assuming that the right hand side is equal to the left hand side in the above relation one gets a transcendental equation. Exact solutions of such an equation can be expressed by means of the Lambert $W$ function [15]. An asymptotic solution of the equation obtained from the relation (37) is relatively easy to find [16]. The very approximate asymptotic solution, $t_{a s}$, of this equation for $\left(\frac{\mathcal{E}_{\phi}}{\gamma_{\phi}^{0}}\right)>10^{2}$ (in general for $\left(\frac{\mathcal{E}_{\phi}}{\gamma_{\phi}^{0}}\right) \rightarrow \infty$ ) has the form

$$
\begin{aligned}
\frac{\gamma_{\phi}^{0} t_{a s}}{\hbar} \simeq & 8,28+4 \ln \left(\frac{\mathcal{E}_{\phi}^{0}}{\gamma_{\phi}^{0}}\right) \\
& +2 \ln \left[8,28+4 \ln \left(\frac{\mathcal{E}_{\phi}^{0}}{\gamma_{\phi}^{0}}\right)\right]+\ldots .
\end{aligned}
$$

\section{Some generalizations}

To complete the analysis performed in the previous Section let us consider a more general case of $a(t)$. Namely, let the asymptotic approximation to $a(t)$ have the form

$$
a(t) \underset{t \rightarrow \infty}{\sim} \sum_{k=0}^{N} \frac{c_{k}}{t^{\lambda+k}},
$$

where $\lambda>0$ and $c_{k}$ are complex numbers. Note that the asymptotic expansion for $a(t)$ of this or a similar form one obtains for a wide class of densities of energy distribution $\omega(\mathcal{E})[2,4,6,6,9,47]$.

From the relation (39) one concludes that

$$
\frac{\partial a(t)}{\partial t} \underset{t \rightarrow \infty}{\sim}-\sum_{k=0}^{N}(\lambda+k) \frac{c_{k}}{t^{\lambda+k+1}} .
$$

Now let us take into account the relation (11). From this relation and relations (39), (40) it follows that

$$
h_{\phi}(t) \underset{t \rightarrow \infty}{\sim} \frac{d_{1}}{t}+\frac{d_{2}}{t^{2}}+\frac{d_{3}}{t^{3}}+\ldots
$$

where $d_{1}, d_{2}, d_{3}, \ldots$ are complex numbers. This means that in the case of the asymptotic approximation to $a(t)$ of the form (39) the following property 
holds,

$$
\lim _{t \rightarrow \infty} h_{\phi}(t)=0 .
$$

It seems to be important that results (41) and (42) coincide with the results (27) - (30) obtained for the density $\omega(\mathcal{E})$ given by the formula (14). This means that general conclusion obtained for the other $\omega(\mathcal{E})$ defining unstable states should be similar to those following from (27) - (30).

\section{Final remarks.}

Recently in many papers it was reported that some results of a detailed analysis of the spectra of very distant astrophysical object could be explained by the variation of time of the fine structure constant $\alpha$ [19, 21, 22, 23]. The analysis of the methods allowing for such an interpretation of the observed spectra shows that the conclusion that $\alpha$ varies in time was drawn by comparing the emission lines of two states separated by the fine-structure interactions and obtained by observing an astrophysical object with the corresponding lines coming form the laboratory source [22]. In the light of the results obtained in Sec. 2 and 3 the question arises if such an interpretation of these astronomical observations is correct: For the observer the effect described by relation (33) and caused by long time properties of time evolution of unstable states, and the effect caused by a hypothetical variation in time of $\alpha$, seems to be indistinguishable. Simply, there is a possibility that the interpretation of the mentioned results of astronomical results may not have taken into account all the available information. In the following we will try to shed some light on the above statement.

So, let us consider a simple toy model. If one identifies energies $\mathcal{E}_{\phi}^{0}$ with energy emitted by an electron jumping from the energy level $n_{j}$ to the energy level $n_{k}$ of the hydrogen atom in the nonrelativistic quantum mechanic then one finds

$$
\mathcal{E}_{n_{j k}}^{0}=-R_{y}\left(\frac{1}{n_{j}^{2}}-\frac{1}{n_{k}^{2}}\right), \quad\left(n_{k}<n_{j}\right),
$$

where $n_{j}, n_{k}=1,2,3, \ldots$ and $R_{y}=\frac{m_{e} e^{4}}{2 \hbar^{2}} \equiv \frac{\alpha^{2} m_{e} c^{2}}{2}$ is the Rydberg constant, $m_{e}$ is the mass of the electron, $c$ is the speed of light and $\alpha$ denotes the fine-structure constant.

Now let these atoms interact with some external fields and let these fields excite the atoms forcing electrons to change their energy levels. Next, let 
these excited hydrogen atoms emit the electromagnetic waves of the energies $\mathcal{E}_{n_{j k}}^{0} \equiv h \nu_{n_{j k}}^{0}$ (where $\nu_{n_{j k}}^{0}$ denotes the frequency of the emitted wave) and let them move away from the observer with the velocity $v$. Then, the observer's measuring devices will show that the energies emitted by these moving atoms are $\mathcal{E}_{n_{j k}}^{0, v}$. The energy $\mathcal{E}_{n_{j k}}^{0}$ emitted by this same source at rest is connected with $\mathcal{E}_{n_{j k}}^{0, v}$ by the Doppler formula [18]

$$
\mathcal{E}_{n_{j k}}^{0, v}=\kappa \mathcal{E}_{n_{j k}}^{0}<\mathcal{E}_{n_{j k}}^{0}
$$

where $\kappa=\frac{1-\beta}{\sqrt{1-\beta^{2}}}$ and $\beta=\frac{v}{c}$, and

$$
\mathcal{E}_{n_{1 k}}^{0, v} \mp \mathcal{E}_{n_{2 k}}^{0, v}=\kappa\left(\mathcal{E}_{n_{1 k}}^{0} \mp \mathcal{E}_{n_{2 k}}^{0}\right) .
$$

¿From (44) and (45) it follows that

$$
\frac{\mathcal{E}_{n_{1 k}}^{0, v}}{\mathcal{E}_{n_{2 k}}^{0, v}} \equiv \frac{\mathcal{E}_{n_{1 k}}^{0}}{\mathcal{E}_{n_{2 k}}^{0}}, \quad \text { or } \quad \frac{\nu_{n_{1 k}}^{0, v}}{\nu_{n_{2 k}}^{0, v}}=\frac{\nu_{n_{1 k}}^{0}}{\nu_{n_{2 k}}^{0}},
$$

where $\mathcal{E}_{n_{j k}}^{0, v}=h \nu_{n_{j k}}^{0, v}$, and similarly

$$
\frac{\mathcal{E}_{n_{1 k}}^{0, v}-\mathcal{E}_{n_{2 k}}^{0, v}}{\mathcal{E}_{n_{1 k}}^{0, v}+\mathcal{E}_{n_{2 k}}^{0, v}}=\frac{\mathcal{E}_{n_{1 k}}^{0}-\mathcal{E}_{n_{2 k}}^{0}}{\mathcal{E}_{n_{1 k}}^{0}+\mathcal{E}_{n_{2 k}}^{0}} .
$$

Now, let us assume for a moment that the $\omega(\mathcal{E})$ given by the formula (14) describes the considered sources with the sufficient accuracy. Next if one additionally assumes that in the distant past photons of energies, say $\mathcal{E}_{n_{j k}}^{0},(j=1,2)$, were emitted and that the sources of this emission were moving away from the observer with known constant velocity $v$, then at the present epoch this observer detects energies $\mathcal{E}_{n_{j k}}^{\infty \prime}=\kappa \mathcal{E}_{n_{j k}}^{\infty}$, where $\mathcal{E}_{n_{j k}}^{\infty} \stackrel{\text { def }}{=}$ $\Re\left(\left.h_{n_{j k}}(t)\right|_{t \rightarrow \infty}\right.$. If the moment of this emission was at suitably distant past then according to the results of Sec 2 (see (35)) it should appear that

$$
\left|\mathcal{E}_{n_{1 k}}^{\infty \prime}-\mathcal{E}_{n_{2 k}}^{\infty \prime}\right|<\left|\mathcal{E}_{n_{1 k}}^{0, v}-\mathcal{E}_{n_{2 k}}^{0, v}\right|
$$

If this observer knows nothing about the effect described in Sec. 2 and 3 and if he also finds that in addition

$$
\frac{\mathcal{E}_{n_{1 k}}^{\infty}}{\mathcal{E}_{n_{2 k}}^{\infty}} \neq \frac{\mathcal{E}_{n_{1 k}}^{0}}{\mathcal{E}_{n_{2 k}}^{0}},
$$


or

$$
\frac{\mathcal{E}_{n_{1 k}}^{\infty}-\mathcal{E}_{n_{2 k}}^{\infty}}{\mathcal{E}_{n_{1 k}}^{\infty}+\mathcal{E}_{n_{2 k}}^{\infty}} \neq \frac{\mathcal{E}_{n_{1 k}}^{0}-\mathcal{E}_{n_{2 k}}^{0}}{\mathcal{E}_{n_{1 k}}^{0}+\mathcal{E}_{n_{2 k}}^{0}} .
$$

then identifying $\mathcal{E}_{n_{j k}}^{\infty \prime}$ with $\mathcal{E}_{n_{j k}, v}^{0,}(j=1,2)$, and making reasonable assumptions that $\frac{1}{\kappa}\left(\mathcal{E}_{n_{1 k}}^{\infty}-\mathcal{E}_{n_{2 k}}^{\infty}\right)$ should also fulfil the relation of the type (43), and the ratio $\frac{\mathcal{E}_{n_{1 k}}^{\infty}}{\mathcal{E}_{n_{2 k}}^{\infty}} \equiv \frac{\mathcal{E}_{n_{1 k}}^{\infty}}{\mathcal{E}_{n_{2 k}}^{\infty}}$ or $\frac{\mathcal{E}_{n_{1 k}}^{\infty}-\mathcal{E}_{n_{2 k}}^{\infty}}{\mathcal{E}_{n_{1 k}}^{\infty}+\mathcal{E}_{n_{2 k}}^{\infty}}$ should be the same as that at the present epoch (see (46)), this observer, on the basis of property (35), is forced to conclude that in the mentioned suitably distant past the Rydberg constant $\left.R_{y}^{\text {past }} \stackrel{\text { def }}{=} R_{y}\right|_{t \rightarrow \infty}$ was smaller than at the present epoch.

On the other hand, if the observer knows the effect described in the previous Sections then he could attribute the decrease of $R_{y}$ in the distant past to the long time behavior of the survival probability $a(t)$ and resulted from this the decrease of the effective Hamiltonian $h_{\phi}(t)$ for $t \rightarrow \infty$ (see (10), (27), (28) and (41) ) rather than to an actual change in the fine structure constant. The conclusion that such an observation can be considered as a confirmation of the mentioned effect seems to be justified. Note that from (46), (33) it follows that this effect seems to be distinguishable from the Doppler effect.

Unfortunately, for the reasons mentioned in Sec. 1 it seems to be rather impossible to observe the discussed effect in laboratory tests. A similar conclusion follows from the relations (37), (38). Nevertheless, this effect should be detectable by means of the observation of spectra of distant astrophysical object.

As mentioned earlier some astrophysical observations suggest that relations of type (48) and (50) take place. More precisely, these observations suggest that at very distant past the fine structure constant $\alpha$ was smaller than at the present epoch (for the recent data see [19]) which implies that $R_{y}^{\text {past }}<R_{y}$. The known physical effects responsible for the observed redshift are unable to explain such a result [20]. Therefore, the hypothesis that $\alpha$ varies in time, $\alpha=\alpha(t)$, and that at the very distant past $\alpha(t)$ was smaller than $\alpha(0)$ (where $\alpha(0)$ denotes the value of $\alpha(t)$ at the present epoch) was formulated (see [19, 21, 22] and references one can find therein). The scale of changes of $\alpha$ is usually described by means of the quantity $\frac{\Delta \alpha(t)}{\alpha(0)}$, where: $\Delta \alpha(t)=\alpha(t)-\alpha(0)$.

One of the methods allowing to estimate $\frac{\Delta \alpha(t)}{\alpha(0)}$ consists in the analysis of the emission lines of two states separated by the fine-structure interactions. 
In such a case to a very good accuracy (see formula (5) in [22])

$$
\left(\frac{\alpha(t)}{\alpha(0)}\right)^{2} \simeq\left[\frac{\lambda_{2}(t)-\lambda_{1}(t)}{\lambda_{2}(t)+\lambda_{1}(t)}\right]\left[\frac{\lambda_{2}(0)+\lambda_{1}(0)}{\lambda_{2}(0)-\lambda_{1}(0)}\right],
$$

where $\lambda_{j}(t),(j=1,2)$, are wavelengths at time $t$. Using the relation $\mathcal{E}_{n_{j k}}(t)=$ $\frac{h c}{\lambda_{j}(t)},(j=1,2)$, one can rewrite this relation as follows

$$
\begin{aligned}
\left(\frac{\Delta \alpha(t)}{\alpha(0)}+1\right)^{2} \simeq & {\left[\frac{\mathcal{E}_{n_{1 k}}(t)-\mathcal{E}_{n_{2 k}}(t)}{\mathcal{E}_{n_{1 k}}(t)+\mathcal{E}_{n_{2 k}}(t)}\right] \times } \\
& \times\left[\frac{\mathcal{E}_{n_{1 k}}(0)+\mathcal{E}_{n_{2 k}}(0)}{\mathcal{E}_{n_{1 k}}(0)-\mathcal{E}_{n_{2 k}}(0)}\right]
\end{aligned}
$$

Taking $t \rightarrow \infty$ in this formula and replacing $\mathcal{E}_{n_{j k}}(t),(j=1,2)$, by relations of type (12), (28), i. e., by $\mathcal{E}_{n_{j k}}^{\infty}$, one obtains an expression for $\left.\frac{\Delta \alpha(t)}{\alpha(0)}\right|_{t \rightarrow \infty}$ connecting the variation of $\alpha$ with the effect described in Sec. 2. Of course the contribution of this effect into the possibly non-zero value of $\frac{\Delta \alpha(t)}{\alpha(0)}$ is only connected with the long time behavior of the survival amplitude $a(t)$, that is with the long time properties of the real part of the effective Hamiltonian, $\mathcal{E}_{n_{j k}}(t)=\Re\left(h_{n_{j k}}(t)\right)$, described earlier and does not signify the change of $\alpha$ with time.

Note that from (33) and other results of Sec. 2 it follows that the right hand side of (52) should not be equal to 1 for $t \rightarrow \infty$. So if one tries to estimate possible variations of $\alpha$ in time $t$ using data obtained from astrophysical observation of a very distant cosmic object, one should take into account that a significant contribution to $\Delta \alpha$ obtained in this way can be a contribution generated by the long-time effect discussed in Sec 2.

Unfortunately, the model considered in Sec. 2 does not reflect correctly all properties of the real physical system containing unstable states. Therefore, formula (33) obtained within this model can not be considered as universally valid and it does not lead to the expression for $\frac{\Delta \alpha(t)}{\alpha(0)}$ correctly describing real properties of very distant astronomical sources of electromagnetic radiation. The defect of this model is that some quantities calculated within it are divergent. Indeed from (3), (5) and (11) one finds that

$$
\left.i \hbar \frac{\partial a(t)}{\partial t}\right|_{t=0}=\langle\phi|H| \phi\rangle,
$$

but inserting into (5) the density $\omega(\mathcal{E})$ given by (14), relation (53) yields $\langle\phi|H| \phi\rangle=\infty$. For this same reason $h_{\phi}(t)$ and $\Delta h_{\phi}(t)$ computed for $\omega(\mathcal{E})$ 
given by (14) are divergent at $t=0: h_{\phi}(=0)=\infty, \Delta h_{\phi}(t=0)=\infty$. Nevertheless, taking into account that a very wide class of models of unstable states leads to a similar asymptotic long time behaviour of $a(t)$ to this obtained for the model considered in Sec. 2, some general conclusions following from the results of Sec. 2 and 3 seem to deserve more attention.

So, the following conclusion which can be drawn from (33) seems to hold: If one considers the same pairs of spectral lines (see formula (92) in [21], or (5) in [22]) then the above discussed contribution into $\Delta \alpha$ calculated with the use of astrophysical data obtained for suitably distant two different sources, such that the second source is much older (i.e. much more distant) than the first one, can be the same for these different sources. What is more (see (33) ), this contribution need not depend on time $t$ but it should depend on such parameters describing the excited energy levels of atoms emitting the registered electromagnetic radiation and corresponding to measured spectral lines as energies $\mathcal{E}_{n_{j k}}^{0}$ and decay rate $\gamma_{n_{j k}}^{0}$ (or lifetimes). This means that estimations of $\Delta \alpha$ performed by means of relations of type (51 - OK), (52) and using the astrophysical data obtained for the given cosmic source emitting the electromagnetic radiation can lead to different values of $\Delta \alpha$ depending on the pairs of spectral lines used for calculations. If the hypothesis that $\alpha$ varies in time is true then it seems to be obvious that the above discussed contribution into $\Delta \alpha$ should be absent when one tries to estimate possible variations of $\alpha$ in time in laboratory test. Therefore one should expect the values of $\Delta \alpha$ obtained in laboratory tests [21] can differ from those obtained using astrophysical data. Simply the magnitude of possible variations of $\alpha$ obtained within the use of astrophysical data can be enhanced by the contribution of the effect discussed in this paper.

All the above remarks concerning estimations of $\Delta \alpha$ using results of observations of the spectra of distant astrophysical objects hold also when one tries to indicate a possible cosmological variation of the proton-electron mass ratio analyzing spectra of the same objects [21, 23]. Cosmic distances and other parameters computed from the observed redshift of very distant objects emitting electromagnetic radiation [20] are calculated without taking into account the possible quantum long time energy redshift described in Sec. 2 and Sec. 3, so these distances as well as values of these parameters need not reflect real values of these parameters and thus need not reflect correctly real properties of the Universe.

At this point it should probably be made clear that the discussion of this 
Section and the results obtained in Sec. 2 and 3 are not meant to provide an alternative explanation of the source of the potential variation of $\alpha$. Rather we point out there is a possibility to go beyond the standard interpretation of some results of the measurements of the scale of this potential variation.

\section{References}

[1] S. Krylov, V. A. Fock, Zh. Eksp. Teor. Fiz. 17, (1947), 93.

[2] L. A. Khalfin, Zh. Eksp. Teor. Fiz. 33, (1957), 1371 [Sov. Phys. — JETP 6, (1958), 1053].

[3] R. E. A. C. Paley, N. Wiener, Fourier transforms in the comlex domain, American Mathematical Society, New York, 1934.

[4] M. L. Goldberger, K. M. Watson, Collision Theory, Willey, New York 1964.

[5] R. G. Newton, Scattering Theory of Waves and Particles, Springer, New York 1982.

[6] L. Fonda, G. C. Ghirardii and A. Rimini, Rep. on Prog. in Phys. 41, (1978), 587.

[7] A. Peres, Ann. Phys. 129, (1980), 33.

[8] P. T. Greenland, Nature 335, (1988), 298.

[9] D. G. Arbo, M. A. Castagnino, F. H. Gaioli and S. Iguri, Physica A 227, (2000), 469.

[10] K. Urbanowski, Phys. Rev. A 50, (1994), 2847.

[11] J. M. Wessner, D. K. Andreson and R. T. Robiscoe, Phys. Rev. Lett. 29, (1972), 1126.

[12] E. B. Norman, S. B. Gazes, S. C. Crane and D. A. Bennet, Phys. Rev. Lett. bf 60, (1988), 2246.

[13] K. M. Sluis, E. A. Gislason, Phys. Rev. A 43, (1991), 4581. 
[14] Handbook of Mathematical Functions, Natl. Bur. Stand. Appl. Math. Ser. No 55, eds. M. Abramowitz nad I. A. Stegun (U.S. GPO, Washington, D.C., 1964).

[15] R. M. Corless, G. H. Gonet, D. E. G. Hare, D. J. Jeffrey and D. E. Khnut, Adv. Comput. Math. 5, (1996), 329.

[16] F. W. J. Olver, Asymptotics and special functions, Academic Press, New York, 1974.

[17] F. M. Dittes, H. L. Harney and A. Müller, Phys. Rev. A 45, (1992), 701.

[18] W. Heitler, The Quantum Theory of Radiation, Oxford University Press, London 1954 and Dover Publications, New York 1984.

[19] J. K. Webb, M. T. Murphy, V. V. Flaubaum, V. A. Dzuba, J. D. Barrow, C. W. Churchill, J. X. Prochaska and A. M. Wolfe, Phys. Rev. Lett. 87, (2001), 091301; J. Magueijo, J. D. Barrow, H. B. Sandvik, Phys. Lett. B 549, (2002), 284; M. T. Murphy, J. K. Webb, V. V. Flaubaum, Mon. Not. R. Astron. Soc. 345, (2003), 609; R. Srianand, H. Chand, P. Petijean and B. Brasil, Phys. Rev. Lett. 92, (2004), 121302; E. Peik, B. Lipphardt, H. Schneider and Chr. Tamm, Phys. Rev. Lett. 93 (2004), 170801; C. J. A. P. Martins, A. Melchiorri, G. Rocha, R. Trotta, P.P. Avelino and P. T. P. Viana, Phys. Lett. B 585, (2004), 29; P. Tzanavaris, J. K. Webb, M. T. Murphy, V. V. Flaubaum and S. J. Curran, Phys. Rev. Lett. 95 (2005), 041301.

[20] E. W. Kolb, M. S. Turner, The Early Universe, Addison-Wesley Publ. Co., 1993.

[21] J.-P. Uzan, Rev. Mod. Phys. 75, (2003), 403.

[22] J. N. Bahcall, Astrophys. J. 600, (2004), 520.

[23] E.Reinhold, R.Buning, U. Hollenstein, A. Ivanchik, P. Petijean and W. Ubachs, Phys. Rev. Lett. 96, (2006), 151101. 